\title{
A CATALOGAÇÃO COMO ATIVIDADE PROFISSIONAL ESPECIALIZADA E OBJETO DE ENSINO UNIVERSITÁRIO
}

\author{
Dulce Maria Baptista
}

\begin{abstract}
Resumo
A catalogação é considerada em seu duplo aspecto de atividade profissional especializada e objeto de ensino universitário. A partir da mudança de paradigma - do tamanho das coleções ao acesso à informação - ocorrida no século XX, analisam-se os desdobramentos e perspectivas da catalogação em função dos avanços tecnológicos, bem como da abrangência da formação universitária e da necessidade constante de treinamento. Diante das dificuldades do ensino da disciplina, e em face das mudanças apontadas, torna-se necessário, entre outros aspectos, um equilíbrio bem dosado entre fundamentação e prática.
\end{abstract}

\section{Palavras-Chave}

Catalogação; Mudança Paradigmática; Normas; AACR; Tecnologias da Informação e da Comunicação; Metadados

\section{ANTECEDENTES}

A história da catalogação contém uma série de fatos e acontecimentos que, paralelamente à própria evolução humana e aos progressos tecnológicos, demonstram que a atividade de catalogar impulsionou o desenvolvimento das bibliotecas, na medida em que é nelas que tradicionalmente se preservam os registros do co- nhecimento, e esses registros demandam, desde sempre, uma organização que assegure sua identificação e localização.

Dos antigos papiros aos livros, ao advento da literatura periódica, os materiais foram se diversificando cada vez mais, o que determinou, por outro lado, a necessidade constante de aperfeiçoamento nas práticas de catalogação. A proliferação de 
regras ao longo do tempo, culminando na criação das Regras de Catalogação AngloAmericanas (AACR), já no século $X X$, refletem bem essa necessidade.

Tornou-se clara também, a percepção de que a catalogação é uma atividade especializada, a ser desempenhada por profissionais. A esse propósito, é interessante observar que, embora a atividade documental tenha existido desde a Antigüidade, ela foi se tornando mais complexa com o tempo, como conseqüência da diversificação do conhecimento humano e dos seus registros, que acabou por impulsionar fortemente o setor editorial em todo o mundo. Como reflexo dessa evolução, as bibliotecas aumentaram de tamanho e as coleções cresceram exponencialmente. Tal crescimento, em seu turno, foi muitas vezes resultante da concepção, vigente durante longo período, da biblioteca como guardiã do conhecimento. Como se sabe, o tamanho do acervo constituiu por muito tempo o principal fator de status da biblioteca.

O que se pretende destacar nesse quadro é o fato de que a atividade de catalogar antecedeu o surgimento da pro-fissão do bibliotecário, como tal, e que esta precedeu a existência de cursos universitários voltados à sua formação específica. Em outras palavras, o que se constata é que a necessidade de conhecimentos especiali- zados foi o que determinou não só a consolidação da profissão, como também a existência de um ensino superior que formasse profissionais habilitados a organizar e disponibilizar todo o imenso estoque de informação registrado e armazenado nas bibliotecas e nos mais variados suportes.

A catalogação, entendida como atividade típica de biblioteca, foi desde logo incluída nos currículos como disciplina obrigatória dos cursos de Biblioteconomia. Já o cenário contemporâneo se caracteriza pela indissociabilidade entre informação e tecnologia. $O$ presente artigo analisa a atividade e o ensino da catalogação nesse contexto.

\section{A MUDANÇA DE PARADIGMA E SEUS DESDOBRAMENTOS}

A idéia de que o acesso à informação é mais importante que o tamanho das coleções de livros contidas numa biblioteca constituiu a principal mudança de paradigma ocorrida no século passado, no que se refere à organização e disponibilização da informação. Tal mu-dança, advinda do entendimento de que a informação se converteu em requisito indispensável à democratização do conhecimento e ao progresso das nações, foi impulsionada, por sua vez, e de forma dramática, pelos avanços tecnológicos ocorridos na área das comunicações e do processamento automático de 
dados. A esse propósito, vale sempre lembrar a famosa constatação de McLuhan (1968) de que o mundo se transformara numa "aldeia global". Essa "aldeia" acabaria criando as condições para o surgimento da chamada sociedade da informação (MASUDA, 1980), calcada em utilização intensiva de tecnologia, de modo que não só a mídia - com o apelo da imagem e a divulgação de notícias em tempo real -, como também as universidades, centros de pesquisa e bibliotecas em todo o mundo, se beneficiariam dos avanços tecnológicos para disseminar, em escala crescente, informações científicas, especializadas, técnicas e artísticas, de forma eficiente e rápida, para fins de estudo, pesquisa, educação e cultura.

Nos ambientes organizacionais de empresas e órgãos governamentais, por outro lado, a informação foi assumindo caráter de instrumento indispensável à tomada de decisão e à vantagem competitiva. Esses aspectos contribuíram não com a ampliação no conceito de biblioteca - de guardiã do conhecimento a geradora de produtos e serviços - como também com o surgimento de novas designações para a organização provedora de informação, tais como centro de documentação, unidade de informação, centro de pesquisa, entre outras - juntamente com a diversificação nos próprios tipos de bibliotecas existentes $-\mathrm{e}$, mais recentemente, bibliotecas virtuais e repositórios informacionais. Foi, inclusive, sob o signo da tecnologia que a biblioteca passou a ser vista como um sistema automatizado de informação, no qual o antigo "leitor" transformou-se em "usuário", e o bibliotecário adquiriu o status de profissional da informação - com novas e complexas atribuições.

\section{A CATALOGAÇÃo COMO ATIVIDADE PROFISSIONAL ESPECIALIZADA}

Mey (1995) e Robredo (2005) descrevem em detalhe a evolução e os rumos que a catalogação foi tomando ao longo dos anos, como atividade tipicamente documentária, ao se ocupar do registro bibliográfico dos documentos, enquanto suportes de informação e, sucessivamente, como objeto de adaptação aos novos tempos, em que ao imperativo do controle bibliográfico somou-se o do intercâmbio de informações. O surgimento de catálogos coletivos, redes cooperativas e sistemas especializados é descrito por esses autores como conseqüência natural e resultado palpável dos avanços tecnológicos. A esse respeito, e de forma esquemática, é possível identificar os seguintes marcos na evolução da catalogação: 
$\rightarrow$ utilização da ficha impressa padronizada $\rightarrow$ invenção do computador $\rightarrow$ advento da internet

No cenário atual, em que a Internet rompeu barreiras físicas e geográficas no que tange à circulação e disponibilização da informação, a atividade específica da catalogação passa a fazer parte de um processo mais amplo e complexo. A crescente introdução de padrões que procuram adequar a organização da informação às novas realidades, a publicação de guias, tutoriais, e de estudos que apontam tendências e perspectivas da catalogação no século $\mathrm{XXI}$, constituem reflexo inequívoco dessa evolução. Os MARC Standards da Library of Congress (2006) e o IFLA Universal Bibliographic Control and International MARC Core Programme (UBCIM), bem como os Metadata Standards and Cataloguing Guidelines dos National Archives do Reino Unido (2002), constituem apenas três exemplos dessas orientações, que se encontram, inclusive, disponíveis na rede, em sua íntegra.

Dentre os estudos que discutem os novos caminhos e identificam tendências, podem ser citados, entre outros, os artigos: Metadata: cataloging by any other name... (MILSTEAD; FELDMAN, 1999); Developments in Cataloging and Metadata (HYATT, 2003); Metadata and cataloging practices (EL-SHERBINI; KLIM, 2004); $A$ bibliographic metadata infrastructure for the twenty-first century (TENNANT, 2004). A esse respeito, merecem destaque dois relatórios: $A$ White Paper on the Future of Cataloging at Indiana University (BYRD et al., 2006) elaborado pela equipe denominada Grupo Tarefa sobre o Futuro da Catalogação (Task Group on the Future of $\mathrm{Ca}$ taloging), e o relatório final preparado para a Library of Congress, tendo como título The Changing Nature of the Catalog and its Integration with Other Discovery Tools (CALHOUN, 2006).

Os Functional Requirements for Bibliographic Records - FRBR (Requisitos Funcionais para Registro Bibliográfico) merecem referência especial por constituírem uma nova proposta em termos teóricos. Ainda em discussão, principalmente no âmbito da IFLA, seu objetivo é funcionar como base conceitual para futuras regras de catalogação. A partir da identificação e especificação de entidades e relacionamentos, o propósito dos FRBR é tornar os registros mais coerentes e úteis aos usuários. O texto referente à apresentação de Ibarra (2005) no I Encuentro Internacional de Catalogadores realizado no final de 2005 em Lima, Peru, é bastante informativo sobre o assunto. O que se conclui, grosso modo, é que há uma busca generalizada por simplificação e objetividade nas práticas da catalogação. 
Coleman (2002), por exemplo, considera a catalogação como um dos processos vinculados à organização do conhecimento. A descrição bibliográfica, instrumentalizada por normas, regras, padrões e formatos, procura não só dar conta da diversidade de suportes, como funcionar como representação mesma do documento - tanto em seus aspectos puramente bibliográficos como também nos aspectos textuais, contemplando, inclusive, a carga semântica contida em cada unidade documentária considerada. Observe-se ainda, que essa unidade documentária, seja ela impressa, eletrônica ou virtual, converteuse em recurso/objeto informacional a ser registrado de forma padronizada, de modo a favorecer a recuperação e o intercâmbio de informações em velocidade praticamente instantânea, e em escala planetária. Todos esses aspectos determinam o surgimento de novas formas de se entender e tratar a informação. E é nesse contexto que o bibliotecário assume, para a autora acima citada, uma série de novos e desafiantes papéis: bibliotecário de catálogo; bibliotecário de metadados; gerente da bases de dados; arquiteto da informação; indexador; webdesigner; gerente do conhecimento; designer de informação. ${ }^{1}$

Em alguns países parece consolidarse uma tendência a relacionar o estudo da arquitetura da informação e da organização do conhecimento em cursos de pósgraduação, tanto lato como stricto sensu, como é o caso das universidades de Wisconsin-Milwakee, Washington e Kent (2006). Ao mesmo tempo, novas publicações periódicas (JOURNAL, 2001) e enciclopédias virtuais acompanham a drástica evolução da gestão, da modelagem e do uso da informação em meio digital. Nesse quadro, catalogação e indexação - entenda-se a descrição física e temática dos documentos nos mais diversos suportes associam-se à usabilidade da informação digital, metadados, taxonomias, modelagem de dados e conteúdos, desenho de bancos de dados, recuperação e descoberta de recursos informacionais (resource discovery).

O que se verifica é que em função de todos esses desdobramentos, o antigo conceito de catalogação - restrito à descrição - evoluiu para o de representação, e representação sempre com vistas ao uso e intercâmbio de todo e qualquer recurso informacional. Por outro lado, a coexistência de recursos eletrônicos e impressos, já no início do século $\mathrm{XXI}$, tem dado lugar a quatro idéias, apontadas por Taylor (2000) como equivocadas, em seu estudo sobre a evolução, o estágio atual e as perspectivas da catalogação. São essas:

\footnotetext{
${ }^{1}$ Tradução literal a partir do texto original. 
1) Os materiais impressos irão desaparecer, e os motores de busca pro-verão acesso à informação eletrônica;

2) As bibliotecas não mais necessitam de catalogadores, e os cursos de Biblioteconomia não precisam ensinar catalogação;

3) As AACR2 são detalhadas em excesso, o que torna necessária a criação de métodos mais simples;

4) Não há necessidade de cabeçaIhos de assunto - a busca por palavrachave é suficiente.

Para ela, os materiais impressos não irão desaparecer em futuro próximo e os motores de busca somente proverão acesso não certificado (non-authority controlled) a alguma informação eletrônica. As bibliotecas precisam de catalogadores, sim, e os cursos de biblioteconomia precisam ensinar os princípios da catalogação, tenham estes o nome que tiverem. As AACR2 são detalhadas, porém baseadas em princípios bastante adaptáveis, e a necessidade de se trabalhar com cabeçalhos de assuntos é uma realidade, já que a busca por palavrachave não the parece suficiente. $A$ autora conclui sua argumentação indagando se o Dublin Core, ou outros metadados, deveriam substituir a catalogação tradicional, e, embora admitindo a eventual necessidade de se ter uma designação diferente para o termo "catalogação", afirma que os princípios básicos de descrição e acesso são reais e precisam ser mantidos.

\section{TREINAMENTO VERSUS FORMAÇÃO ACADÊMICA}

Em sua dimensão de atividade técnica especializada, a catalogação possui desde sempre uma característica de prática cotidiana visível, que consiste basicamente no registro - hoje em dia, na inclusão dos registros num sistema automatizado - visando a identificação, localização e recuperação de documentos. A utilização integrada das AACR e do formato MARC, por exemplo, possibilitada pela variedade de programas de computador que a tecnologia coloca no mercado, cria, por sua vez, uma necessidade constante de treinamento por parte dos bibliotecários, de modo a que possam tirar o máximo proveito dos novos recursos e ferramentas de trabalho.

Paralelamente, ocorre que em complementação à formação propriamente acadêmica, o dia-a-dia da catalogação se baseia concretamente nos códigos, em catálogos internos, listas de autoridades, cabeçalhos de assunto, consulta aos catálogos centralizados via Internet (como, por exemplo, os catálogos da Biblioteca Nacional e os da Library of Congress), e nos manuais. A esse respeito, vale citar, inclusive, os manuais elaborados por Mey 
(2003) e Ribeiro (2004) no Brasil, que buscam justamente facilitar o trabalho do catalogador, garantindo-lhe os padrões de qualidade que resultem em consistência e confiabilidade dos registros. Mencionem-se também as publicações de Fecko (1993), Weber (2002), Fritz e Fritz (2003), Gorman (2004), que, entre tantas outras, se preocupam com o passo-a-passo da descrição bibliográfica, tanto em função da variedade de suportes da informação, como também da complexidade do seu tratamento.

A constatação que se torna praticamente inevitável é que, a par da formação acadêmica, a catalogação demanda atualização constante e treinamento contínuo. $\mathrm{E}$ esse treinamento se dá, via de regra, nos ambientes de trabalho, e não necessariamente na universidade, na medida em que é neles que se colocam em prática as formas de organizar a informação que efetivamente atendem os objetivos institucionais de cada tipo de organização. $E$ isso, tendo em vista as particularidades de cada uma, seja com relação ao seu público alvo, seja com relação aos recursos humanos e à capacitação tecnológica de que necessita, e principalmente em função dos recursos orçamentários que the permitam dispor, ou não - considerando-se, por exemplo, o contexto das bibliotecas brasileiras dos aplicativos ou ferramentas mais avançados existentes no mercado.
Tal situação, por outro lado, parece dar origem a uma dicotomia artificial, ou seja, aquela na qual o ensino se torna insuficiente ou obsoleto frente à realidade, enquanto que o treinamento habilita os profissionais a enfrentarem os desafios que se apresentam. Em um artigo esclarecedor sobre o assunto, Hill (2004) questiona até que ponto o ensino da catalogação se tornou obsoleto, desapareceu, ou se transformou. Observa que aquilo que os catalogadores aprendiam originalmente, tanto como alunos dos cursos de Biblioteconomia, como na condição de aprendizes em seus empregos, era suficiente para toda a carreira profissional. Posteriormente, com os progressos verificados na área, o ensino foi se tornando cada vez mais fragmentário. A catalogação, em si, tornou-se inegavelmente mais complexa, com a rápida evolução de formatos, padrões, funções, opções de exibição e busca, bem como com algumas mudanças fundamentais nas expectativas referentes ao controle bibliográfico. Não obstante, as escolas tentam adequar a formação nessa área a uma grade curricular considerada suficiente há cerca de sessenta anos. A tendência hoje em dia, diz a autora, é a de se substituir cursos iniciais de catalogação por um curso mais geral de organização da informação. Como reflexo dessa tendência, e ainda que uma das disciplinas optativas mais populares se refira a recursos não tradicio- 
nais, alguns professores consideram contra-indicada a segmentação de cursos por tipo de material, já que, segundo eles, é preciso preparar o aluno para lidar com todos os tipos de suportes.

Hill reconhece que a formação que os catalogadores recebem constitui apenas um ponto de partida e inicia a segunda parte de seu artigo, observando que nenhum catalogador completa sua formação na universidade. Os programas de Biblioteconomia e Ciência da Informação (Library and Information Science, nos Estados Unidos) tendem a estabelecer uma distinção entre formação e treinamento, e que muitos não consideram apropriado que os cursos de graduação se envolvam em treinamento. A justificativa é que o treinamento visa ao aprendizado sobre a prática específica da biblioteca e sobre processos detaIhados, enquanto que a formação se preocupa com a história, o raciocínio fundamentado (rationales), análise e princípios subjacentes.

Na perspectiva da educação continuada, por outro lado, Hill considera que o ideal é que se possa lançar mão daquilo que entende como "recursos educacionais internos" e "recursos educacionais externos". Em outras palavras, ela identifica a clara necessidade de complementação entre os conhecimentos teóricos e mais abrangentes adquiridos na universidade - os quais se referem à fundamentação e à razão de ser da catalogação -, e os conhecimentos práticos que, nunca suficientes em si, em função de constantes mudanças e novidades típicas da área, demandam treinamento e aperfeiçoamento contínuos no decorrer de toda a vida profissional. Nesse contexto, associações profissionais, redes bibliográficas, simpósios e workshops, bem como a comunidade de catalogadores são alguns dos recursos externos considerados.

De forma mais pontual, certamente como reflexo da consciência de que o ensino precisa desenvolver seus próprios métodos e recursos - independentemente da necessidade sempre presente de treinamento, diversos autores procuram formular propostas, e assim, incorporar novos recursos ao ensino. Tal é, por exemplo, o caso de Lima e Nogueira (2000), ao proporem "A criação de um protótipo hipertextual para instrução do CCAA2".

Moura e Correia (2000) também tratam do hipertexto voltado ao ensino. Saye (2001) estuda a organização de recursos eletrônicos no currículo da biblioteconomia e ciência da informação. Já Hsieh-Yee (2004) faz uma análise pormenorizada da formação em catalogação e metadados, tal como presente nos currículos dos Estados Unidos. 
Ao comparar o que tem ocorrido no mundo e no Brasil com respeito ao ensino da representação descritiva, Mey (2005, p.1) constata que "desde 1997, a catalogação sofre uma reviravolta ao nível internacional", e que o Brasil não tem acompanhado as transformações. A partir de alguns questionamentos, faz sugestões que, no seu entender, poderiam justamente redimensionar esse ensino no país.

\section{CONCLUSÃO}

Tendo em vista a evolução da catalogação a partir do final do século $X X$, e as dificuldades inerentes ao ensino da disciplina nas universidades, torna-se pertinente uma reflexão que contemple os seguintes aspectos:

a) A catalogação, como atividade voltada à identificação, registro e localização de documentos, antecedeu o seu ensino, enquanto disciplina curricular nas universidades;

b) O surgimento formal da profissão de bibliotecário deu-se posteriormente ao advento da catalogação;

c) A formação universitária dos catalogadores tem se mostrado insuficiente em função das mudanças ocorridas na própria natureza da catalogação, mudanças essas resultantes da diversificação nos suportes da informação e dos avanços tecnológicos;

d) Em razão de tais mudanças, o bibliotecário é colocado diante de novos e contínuos desafios, que, inclusive, determinam uma diversificação em seu perfil profissional;

e) A catalogação, como tal, continua sofrendo mudanças em sua natureza e processos, o que determina a necessidade de conhecimento em relação a essas mudanças, e treinamento em relação às práticas delas resultantes;

f) Em função de todas as mudanças apontadas, torna-se necessário alcançar, em nível de currículo, um equilíbrio bem dosado entre fundamentação e prática;

g) Os estágios têm importante contribuição a dar, ao proporcionarem uma percepção correta - e focada nos problemas reais - do mundo em que os futuros profissionais irão atuar, ao tempo que Ihes acrescentam novas habilidades e competências.

Nesse contexto, é forçoso reconhecer que o que parece mais carente de discussão e redimensionamento é o próprio ensino. O treinamento, por ser prático e específico em sua natureza, acaba sendo muitas vezes suficiente para atender às necessi- 
dades em determinado ambiente de trabaIho.

O desafio do ensino universitário, principalmente ao se considerarem as demandas da sociedade, é não se converter num grande e difuso treinamento - como que a reboque dos imperativos do mercado - privilegiando uma abordagem exclusivamente tecnicista em detrimento da discussão e da reflexão crítica, as quais, inclusive, impulsionam a área, e dão origem a mudanças.

\section{REFERÊNCIAS}

BYRD, J. et al. A white paper on the future of cataloging at Indiana University, January 15, 2006. Disponível em:

$<$ http://www.iub.edu/ libtserv/pub/Future_of Cataloging_White_Paper.pdf $>$. Acesso em: 12 nov. 2006.

CALHOUN, Karen. The changing nature of the catalog and its integration with other discovery tools: Final report, March 17, 2006; prepared for the Library of Congress. Disponível em:

$<$ http://www.loc.gov/catdir/calhoun-reportfinal.pdf>. Acesso em: 12 nov. 2006.

COLEMAN, Anita. Intellectual access \& the organization of information. University of Arizona: Aug. 19, 2002. Disponível em: $<$ http://www.sir.arizona.edu/faculty/coleman /sstart02/ascsstrt02.pdf>. Acesso em: 11 nov. 2006.

EL-SHERBINI, Magda; KLIM, George. Metadata and cataloging practices. The Electronic Library, v.22, n.3, P. 238-248, 2004.

FECKO, Mary Beth. Cataloging nonbook resources: a how-to-do-it manual for librari- ans. New York: Neal-Schuman Publishers, c1993.

FRITZ, Deborah H.; FRITZ, Richard J. MARC21 for everyone: a practical guide. Chicago: American Library Association, 2003. $188 \mathrm{p}$.

GORMAN, Michael. The concise AACR2, 2004 revision. Chicago: American Library Association, 2004.

HILL, Janet Swan. Education and training of catalogers: obsolete? disappeared? transformed? Technicalities, v.24 n.1, p. 113, Jan./Feb., 2004.

HSIEH-YEE, Ingrid. Cataloging and metadata education in North American LIS programs. Library Resources \& Technical Services, v.48, n.1, p. 59-68,, Jan. 2004.

HYATT, Shirley. Developments in cataloging and metadata. In: Gorman, G.E.; Dorner, Daniel G. (eds.). International Yearbook of Library and Information Management 2003-2004: Metadata applications and management. London: Facet Publishing, 2003. Disponível em:

<http://www.oclc.org/research/publications/ archive/2003/hyatt.pdf>. Acesso em: 12 nov. 2006.

IBARRA, Ana María Talavera. FRBR: Requerimientos Funcionales de los Registros Bibliográficos. In: CENTRO BIBLIOGRÁFICO NACIONAL (org.). Encuentro Internacional de Catalogadore, 1. 2005, Lima, Peru. Nuevas tendencias en la normalización y sistematización de la información: ponencias y conclusiones. Lima: Biblioteca Nacional del Perú; Fondo Editorial, 2006.

IFLANET. International Federation of Library Associations and Institutions; IFLA Universal Bibliographic Control and international MARC Core Programme (UBCIM). UNIMARC minimal level record. UNIMARC Guidelines, n.4, 1999. Disponível em: $<$ http://www.ifla.org/VI/3/p19961/guid4.htm>. Acesso em: 13 nov. 2006. 
JOURNAL OF DIGITAL INFORMATION. Disponível em: <http://jodi/tamu.edu.> Acesso em: 14 nov. 2006.

KENT State University. Revised Information Architecture and Knowledge Management Curriculum. Disponível em:

<http://iakm.kent.edu>. Acesso em: 27 nov. 2006.

THE LIBRARY Of CONGRESS. Network Development and MARC Standards Office. MARC Standards. Disponível em: <http://www.loc.gov/marc/> Acesso em: 13 nov. 2006.

LIMA, Gersina Ângela Borém; NOGUEIRA, Isabel Cristina. A criação de um protótipo hipertextual para instrução do CCAA2. In: SEMINÁRIO NACIONAL DE BIBLIOTECAS UNIVERSITÁRIAS, 11. 2000, Florianópolis. Anais... Florianópolis: UFSC, 2000. Disponível em: <http://snbu.brs.br/snbu2000/docs/pt/doc/t042.doc>. Acesso em: 12 nov. 2006.

MASUDA, Yoneji. The information society as post-industrial society. Tokyo: Institute for Information Society, 1980.

McLUHAN, Marshall. War and peace in the global village. 1st. ed. New York: Bantam, 1968. Referência disponível em: $<$ http://en.wikipedia.org/wiki/McLuhan\#War and_Peace_-

in_the_Global_Village_281968.29>. Acesso em: 12 nov. 2006.

MEY, Eliane Serrão Alves. Algumas questões sobre o ensino da representação descritiva, ou a catalogação na berlinda. Disponível em: <http://www2.fgv.br/bibliodata/geral/docs/TextoElianeSerraoAlvesMey.pdf > Acesso em: 13 nov. 2005.

MEY, Eliane Serrão Alves. Introdução à catalogação. Brasília: Briquet de Lemos/Livros, 1995. 123 p.

MEY, Eliane Serrão Alves. Não brigue com a catalogação! Brasília: Briquet de Lemos/Livros, 2003
MILSTEAD, Jessica; FELDMAN, Susan. Metadata: Cataloging by any other name... Online, Jan., 1999. Disponível em: <http://www.onlineinc.com/onlinemag/OL19 99/milstead1.html> Acesso em: 12 nov. 2006.

MOURA, Ângela Maria Saraiva de; CORREIA, Anna Elizabeth Galvão Coutinho. Hipertexto para o ensino da representação descritiva: uma experiência de modelagem. Informação \& Sociedade: Estudos, v.10, n. 1, 2000.

THE NATIONAL ARCHIVES. Metadata standards and cataloguing guidelines. Metadata Standards and Cataloguing Rules. Version 2, Oct. 2002 Disponível em: $<$ http://www. nationalarchives.gov.uk/documents/metedata_v2.pdf.> Acesso em: 13 nov. 2006.

RIBEIRO, Antonia Motta de Castro Memória. Catalogação de recursos bibliográficos pelo AACR2. 2 ed. rev. Brasília: Ed. do Autor, 2004.

ROBREDO, Jaime. Documentação de hoje e de amanhã. 4. ed. rev. e ampl. Brasília: Ed. do Autor, 2005.

SAYE, Jerry D. The organization of electronic resources in the library and information science curriculum. OCLC Systems and Services, v.7, n.2, P. 71-78, 2001.

TAYLOR, Arlene G. Cataloging: Ticket to the past, the present, and the future: In: Resources and Technical Services Forum, lowa Library Association Annual Conference, October 20, 2000. Disponível em: <http://slisweb.sjsu.edu/Institute/Taylor/sld0 01.htm> Acesso em: 13 nov. 2006.

TENNANT, Roy. A bibliographic metadata infrastructure for the twenty-first century $\mathrm{Li}$ brary Hi Tech, v.22, n.2, P. 175-181, 2004.

UNIVERSITY of Washington. Information Architecture Summer Institute. UW Extension. Disponível em: <http://www.extension.washington.edu/ext/ 
special/infoarch> Acesso em: 27 nov. 2006.

UNIVERSITY of Wisconsin-Milwaukee. School of Informatiom Studies. Information Architecture and Knowledge Organization: Tentative Draft Syllabus. Disponível em: $<$ http://www.uwm.edu/People/mll/791InfoArch.htm>. Acesso em: 27 nov. 2006.

WEBER, Mary Beth. Cataloging nonprint and internet resources: a how-to-do-it manual for librarians. New York: NealSchuman Publishers, c2002.

Dulce Maria Baptista

Professora Adjunta do Departamento de Ciência da Informação e Documentação da UnB. Licenciada em Letras (PUC/RJ). Bacharel em Biblioteconomia. Mestre em Educação. Doutora em Ciência da Informação (UnB). dmbp@unb.br

\section{Title}

Cataloging as a specialized professional activity and object of academic learning

\section{Abstract}

Cataloging is considered in its double aspects as a specialized professional activity and as an object of academic learning. Considering the paradigm change - from collection size to information access - that took place in the $\mathrm{XX}$ century, outcomes and prospects in cataloging are analized, having in view the technological advances, as well as the scope of academic education and the need of continuous training in this field. Regarding some limitations in cataloging education and due to the changes that are pointed out, it becomes necessary, among others factors, to find the best balanced education, both in terms of theoretical framework and in terms of practice.

\section{Keywords}

Cataloguing; Paradigm Change; Standards; AACR; Information and Communication Technologies; Metadata; Education; Training.

\section{Título}

La catalogación como actividad profesional especializada y objeto de una asignatura académica

\section{Resumen}

La catalogación es considerada bajo sus dos aspectos de actividad profesional especializada y objeto de enseñanza universitaria. A partir de la mudanza de paradigma - desd el tamaño de las colecciones al acceso a la información ocurrida en el siglo XX, se analisan los desdoblamientos y perspectivas de la catalogación en función de los avances tecnológicos, así como de la amplitud de la formación universitaria y de la necesidad constante de entreinamiento. Ante las dificultades de la enseñanza de la asignatura y de los cambios señalados, se hace necesario, entre otros aspectos, un equilibrio entre fundamentación y práctica.

\section{Palabras Clave}

Catalogación; Mudanza Paradigmática; Normas; AACR; Tecnologías de la Información y de la Comunicación; Metadatos; Educación; Capacitación.

Recebido em: 01.12.2006

Aceito em: 20.12.2006 